\title{
Des lacunes de la promotion de la santé
}

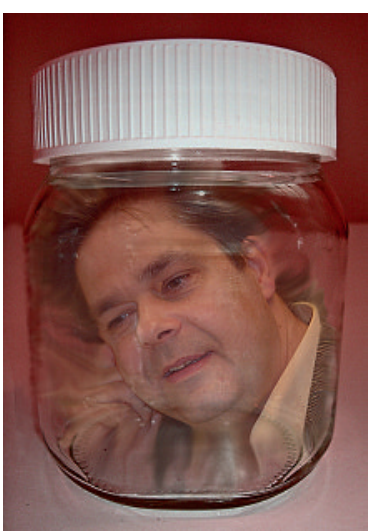

Eberhard Wolff
Je l'avoue: nous donnons du chocolat à nos enfants! Chaque soir! Et même beaucoup de chocolat, s'ils le veulent! Et du coca-cola! Même des chips! Ouf, maintenant je l'ai dit!

Ma confession est due à l'ouvrage polémique de 163 pages sur la promotion de la santé, publié l'année dernière par Christoph Klotter [1], psychologue diététicien allemand et spécialiste en promotion de la santé, lequel n'a pas pris la plume pour traiter de mythes les débats sur l'embonpoint et la mauvaise alimentation [2], leur reprocher de générer des mouvements de panique [3] ou même de mentir [4]. Laissons de côté la question de savoir si le chocolat, le coca-cola et les chips nuisent vraiment à la santé. Ce livre donne d'autres explications sur la raison pour laquelle nous donnons du chocolat à nos enfants et faisons partie de l'immense majorité des gens qui ont rendu nécessaire l'élaboration de programmes onéreux pour la prévention de la santé et le conseil diététique, programmes dont le succès est finalement mis en doute. Klotter se penche sur l'efficacité et la légitimité de la forme prise aujourd'hui par la prévention de la santé, ses échecs et leurs causes, d'où aussi son titre: «Pourquoi réussissons-nous à ne pas rester en bonne santé?» (trad.).

Mues par les meilleures intentions du monde, les personnes qui gardent nos enfants les soumettent à la loi du «manger sainement» sans leur laisser vraiment le choix. Klotter est d'avis que la promotion de la santé est conçue globalement sur le même schéma. Elle ne demande pas aux gens ce qu'ils veulent manger, mais elle part du principe qu'elle sait ce qui est bon pour eux. Klotter appelle cela un déficit de participation et y voit une lacune majeure. Il l'explique par les présupposés de la promotion de la santé concernant l'être humain: elle est l'une des dernières grandes utopies restantes de notre société et - comme d'autres mouvements s'auto-déclarant avant-gardistes - elle croit détenir la vérité absolue avec le mot d'ordre: celui qui intervient en faveur de la santé est du côté du bien. La promotion de la santé ressemble ainsi à un «mouvement de Réveil», elle a une pensée totalitaire im-plicite et se trouve dans un absolutisme éclairé parce qu'elle accepte l'autodétermination aussi longtemps qu'il y a une prise en compte de la santé.

Klotter estime donc que la promotion de la santé ne défend aucune vérité dernière mais qu'il s'agit de cette morale spécifique intitulée «éthique protestante» par le sociologue Max Weber. Dit simplement: nous n'existons pas pour jouir mais pour travailler durement et être productifs. Il nomme cela «l'ascèse du monde intérieur». La promotion de la santé vend ainsi l'éthique protestante en tant que raison universelle et elle tente de prescrire ce mode de vie dans le cadre d'une pensée globale.
Pour les gardiennes de nos enfants, «manger sainement» est un objectif très important. Pour nos enfants, «manger sainement» n'est pas le but suprême. Pour eux, manger c'est goûter à quelque chose de bon et non pas suivre l'éthique de la pyramide alimentaire. Selon Klotter, la promotion de la santé a tendance à faire de la santé une valeur absolutiste. Le plaisir et la détente sont importants uniquement s'ils sont utiles à la santé. Mais l'être humain a d'autres besoins à part celui de vivre sainement: paresser, s'adonner à un «vice», «pécher», éprouver de l'ivresse, courir un risque. Georges Bataille appelle cela le besoin fondamental de dépenses improductives. Et pourquoi la «santé» doitelle est toujours être plus importante que la «culture»?

Pendant des années, nos enfants n'ont pas pu s'accommoder à l'ordre de «manger sainement» au quotidien. Klotter pense que c'est dû au fait que les recommandations en la matière sont beaucoup trop liées au contrôle concret du comportement. Même ces conseils avisés contiennent une part de «pouvoir disciplinaire» et de contrôle sur la corporéité, comme Foucault l'a déjà décrit sur la base des prisons et des hôpitaux de la Renaissance. Nos enfants le remarquent aussi. Le résultat: un certain scepticisme, peutêtre aussi le chocolat, le soir à la maison, en dépit des prescriptions diététiques en vigueur. Ils nomment les légumes verts «le poison destiné aux enfants». Le sarcasme agit comme soupape de sécurité. Par ailleurs, ils mangent librement les produits «rouges» et ils sont tout contents lorsque les carottes et les tomates sont savoureuses [5].

Le schéma moraliste du bien et du mal pour la promotion de la santé crée finalement, selon Klotter, aussi des «coupables». Ce qui est normal est bien. Celui qui n'est pas normal risque d'être rejeté. A l'école primaire, les camarades de classe de nos enfants déterminent régulièrement qui est «gros».

Klotter est d'avis qu'une promotion percutante de la santé peut contribuer à diviser la société en personnes axées ou non sur les préceptes de santé, chacun se délimitant par rapport aux autres. Pierre Bourdieu a désigné ce phénomène par la notion de «distinction». Les uns vivent sainement pour montrer qu'ils ne font pas partie des gens «malsains», lesquels se démarquent des premiers pour ne pas faire partie de ceux «qui savent mieux».

L'écrit polémique publié par Klotter est très pointu, souvent théorique. Une lecture attentive montre que de nombreux éléments de notre quotidien s'y reflètent. Et finalement, je découvre régulièrement en moi un morceau bien gras d'éthique protestante.

Eberhard Wolff* 